\title{
Efficacy and Safety of Varenicline for Smoking Cessation in Schizophrenia: A Meta-Analysis
}

\author{
Saeed Ahmed ${ }^{1}$, Sanya Virani ${ }^{2}$, Vijaya P. Kotapati ${ }^{3 *}$, Ramya Bachu ${ }^{4}$, Mahwish Adnan ${ }^{5}$, \\ Ali M. Khan ${ }^{6}$, Aarij Zubair ${ }^{7}$, Gulshan Begum ${ }^{3}$, Jeevan Kumar ${ }^{8}$, Mustafa Qureshi ${ }^{9}$ and \\ Rizwan Ahmed ${ }^{10}$ \\ ${ }^{1}$ Nassau University Medical Center, East Meadow, NY, United States, ${ }^{2}$ Maimonides Medical Center, New York, NY, \\ United States, ${ }^{3}$ Manhattan Psychiatric Center, New York, NY, United States, ${ }^{4}$ Zucker Hillside Hospital, New York, NY, \\ United States, ${ }^{5}$ Department of Cognitive Behavioral Science, McMaster University, Hamilton, ON, Canada, ${ }^{6}$ Department of \\ Psychiatry, University of Texas Rio Grande Valley, Harlingen, TX. United States, ${ }^{7}$ St. John's University, New York, NY, \\ United States, ${ }^{8}$ Bolan Medical College, Quetta, Pakistan, ${ }^{9}$ Texas Tech University Health Sciences Center, Lubbock, TX, \\ United States, ${ }^{10}$ Liaquat National Medical College, Karachi, Pakistan
}

Objective: Smoking represents a major public health problem among patients with schizophrenia. To this end, some studies have investigated the efficacy of varenicline for facilitating smoking cessation in schizophrenia patients. The present review seeks to synthesize the results of these studies as well as document the reported side effects of using this medication.

Edited by:

Marijn Lijffijt,

Baylor College of Medicine,

United States

Reviewed by:

Jinhua Wu,

Florida Atlantic University,

United States

Olga Valverde,

Universidad Pompeu Fabra, Spain

${ }^{*}$ Correspondence:

Vijaya P. Kotapati

padmakotapati89@gmail.com

Specialty section:

This article was submitted to

Psychopharmacology,

a section of the journal

Frontiers in Psychiatry

Received: 21 May 2018

Accepted: 20 August 2018

Published: 19 September 2018

Citation:

Ahmed S, Virani S, Kotapati VP, Bachu R, Adnan M, Khan AM,

Zubair A, Begum G, Kumar J,

Qureshi M and Ahmed R (2018)

Efficacy and Safety of Varenicline for

Smoking Cessation in Schizophrenia:

A Meta-Analysis

Front. Psychiatry 9:428

doi: 10.3389/fpsyt.2018.00428
Methods: An electronic search was performed using five major databases: PubMed, Scopus, EMBASE, Web of Science, and Cochrane Library. Included in the current analysis were randomized clinical trials (RCTs) that have investigated the effect of varenicline in promoting smoking cessation in patients with schizophrenia. Risk of bias among included RCTs was assessed using the Cochrane Collaboration's quality assessment tool.

Results: Among the 828 screened articles, only four RCTs, which involved 239 participants, were eligible for meta-analysis. In patients with schizophrenia, varenicline treatment when compared to placebo significantly reduced the number of cigarettes consumed per day [SMD $(95 \% \mathrm{Cl})=0.89(0.57-1.22)]$ and expired carbon monoxide levels [SMD $(95 \% \mathrm{Cl})=0.50(0.06-0.94)]$ respectively.

Conclusion: Despite a limited number of studies included in the meta-analysis, our results suggest that varenicline is an effective and safe drug to assist smoking cessation in patients with schizophrenia. Future large-scale well-designed RCTs are required to validate these findings.

\section{Keywords: schizophrenia, cigarettes, nicotine addiction, smoking cessation, varenicline}

\section{INTRODUCTION}

Smoking among patients with schizophrenia continues to be a major public health problem. There is a strong association between schizophrenia and smoking behaviors, with prevalence rates ranging from 70 to $90 \%$; as a comparison, smoking rates of the general population in the United States stands at $\sim 30 \%(1,2)$. Also, compared to smokers in the general population, schizophrenic smokers 
consume more cigarettes per day (3). Schizophrenic patients usually smoke heavily, extract higher levels of nicotine, and have higher nicotine dependence scores than smokers who do not have schizophrenia (3-7).

Several hypotheses have been proposed to explain the high rates of smoking in this patient population. The most popular hypothesis, which is known as "self-medication hypothesis (SMH)", argues that these patients smoke in order to manage their negative symptoms by regulating and compensating for the underlying neurobiological deficits associated with the disorder such as cognitive deficits. Smoking may transiently alleviate negative symptoms in schizophrenic patients by increasing dopaminergic and glutamatergic neurotransmission in the prefrontal cortex. Also, nicotine improves some cognitive deficits common in patients with schizophrenia, such as sensory gating deficits, abnormalities in smooth-pursuit eye movements, selective attention, visuospatial working memory limits, and attention deficits; also, high-dose nicotine increases abnormality in P450 inhibition (8). Contrary to these temporary benefits, there is abundant evidence that clearly shows that smoking is harmful because it increases the risk of contracting numerous medical illnesses such as lung cancer and other smoking-related diseases. Mortality rates also reflect this trend; patients with schizophrenia who smoke face mortality rates from smokingrelated health issues that are two to six times higher than other smokers. They confront increased risks of cardiovascular disease, respiratory disease, and cancer (9). The increased mortality risks that result from smoking exacerbates already high mortality risk schizophrenic patients. This higher mortality risk results in about a $20-25 \%$ reduction in average lifespan for people with schizophrenia (4). Schizophrenic patients are at higher risk of fatal cardiovascular disease and other causes of premature death such as obesity, sedentary lifestyle, diabetes, hypertension, or hypertriglyceridemia (10). The risk of HIV (human immunodeficiency virus) and infectious hepatitis is also high in this population. The suicide rate is $10 \%$ compared to $1 \%$ in the general population; the most likely explanations for this higher risk of death include less access to medical care, engagement in high-risk behaviors, and poorer compliance with treatment (11). Concerning morbidity in particular, secondgeneration antipsychotics are more likely to cause weight gain and metabolic syndrome compared to first-generation antipsychotics, and these side effects are associated with a two-to-threefold increase in cardiovascular mortality and a twofold increase in mortality in general (12). The increased risk of mortality in schizophrenics affects men and women equally, although there are sex differences in the epidemiological features of schizophrenia (12). Therefore, the added health risks associated with smoking are particularly dangerous to this population and must be addressed.

Clinicians often do not address nicotine addiction in the schizophrenic population because of the popular beliefs that treatment could worsen the patient's illness or that the patient is not willing to quit (3). However, numerous studies have demonstrated that stable patients with schizophrenia can tolerate cessation attempts without an overall worsening of their illness and can have moderate short-term success in smoking cessation
(2-4, 13-22). Once these patients demonstrate a willingness to quit smoking, they can try numerous therapeutic options that have proven to be beneficial. In 2006, the FDA approved varenicline, a partial agonist of $\alpha 4 \beta 2$ acetylcholine receptor and a full $\alpha 7$ nicotinic acetylcholine receptor agonist for smoking cessation. This drug ameliorates withdrawal symptoms, specifically reducing cravings, and it works as a positive reinforcement for the cessation of smoking. However, there have been some reports of side effects that include the exacerbation of psychiatric symptoms such as depression, aggression, psychosis, and suicidal thoughts and behaviors (23-28). As a result of these sometimes dangerous side effects, the FDA issued a black box warning in 2009 (29). However, several recent studies have provided evidence regarding its safety in facilitating smoking cessation in patients with schizophrenia $(9,15,30-33)$. Numerous published clinical trials have found varenicline to be effective, safe, and well tolerated in terms of neuropsychiatric adverse events, with a common possible side effect of mild to moderate nausea similar to that experienced by the general populations (3, 4, 34-39). A recently published meta-analysis

TABLE 1 | List of studies considered at the final stage of study selection.

\begin{tabular}{lcl}
\hline Study & Included? & Reason for exclusion \\
\hline $\begin{array}{l}\text { Dutra et al. (42) } \\
\text { Williams et al. (33) }\end{array}$ & Yes & No control group \\
Pachas et al. (9) & No & No control group \\
$\begin{array}{l}\text { Fatemi et al. (45) } \\
\text { (clinicaltrial.gov) }\end{array}$ & No & No baseline measures with which to \\
Weiner et al. (19) & Yes & assess outcomes \\
Jeon et al. (43) & Yes & \\
$\begin{array}{l}\text { Coles et al. (32) } \\
\text { (poster abstract) }\end{array}$ & No & No relevant data to extract. \\
& & $\begin{array}{l}\text { Conference poster; only abstract } \\
\text { available }\end{array}$ \\
Hong et al. (46) & Yes &
\end{tabular}

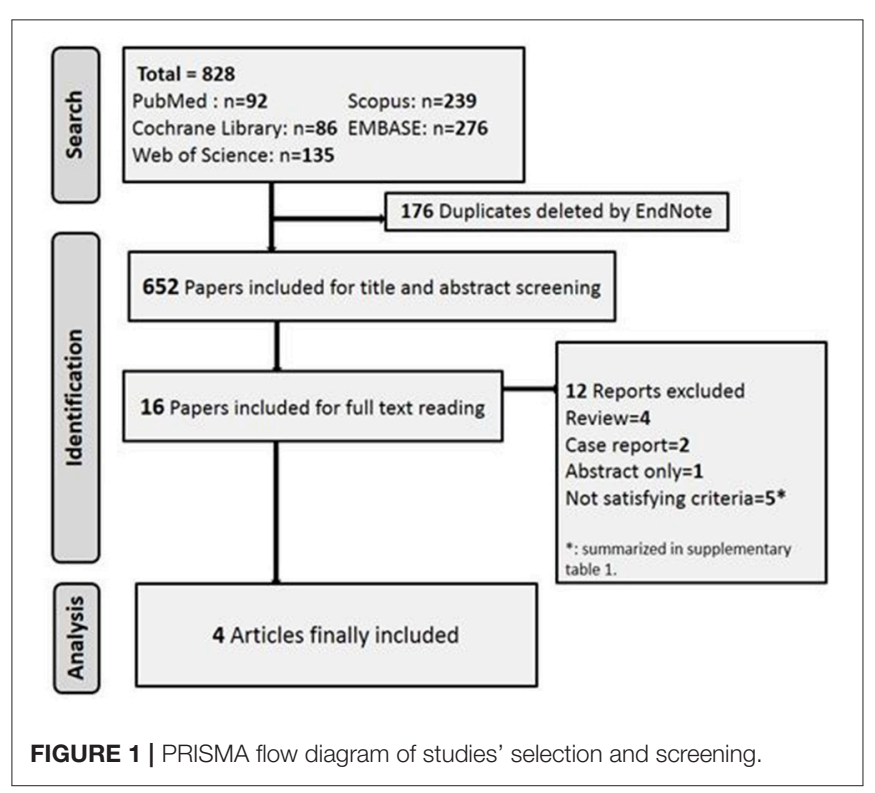


of this subject concluded that varenicline is not superior to placebo in smoking cessation (40). However, the results of this metanalysis run counter to numerous studies that have found varenicline to be an effective, safe, and well-tolerated treatment for smoking cessation in schizophrenic patients $(3,33,41,42)$. Because of these positive findings as well as a new study on this topic (43), we sought to synthesize previous findings and examine the overall effect of varenicline on smoking cessation among patients with schizophrenia.

\section{METHODS}

\section{Search Strategy and Selection Criteria}

The Recommendations from the Preferred Reporting Items for Systematic Reviews and Meta-Analyses (PRISMA) Statement were adopted to conduct this systematic review and metaanalysis (44). Five databases were systematically searched including PubMed, Scopus, EMBASE, Web of Science and Cochrane's library for articles published up until July 2018. We performed an electronic search on this topic with no restrictions regarding language or publication date. The Boolean search we conducted utilized the following keyword combinations: varenicline AND [(schizophrenia spectrum disorder) OR schizophrenia OR (schizoaffective disorder) OR (schizophreniform disorder) OR (delusional disorder)], the strategy was adjusted to suit each of the chosen search engines and databases. We further considered performing a manual search in reference lists of relevant studies. Once we completed the search, we elected to include only RCTs that investigated the effects of varenicline on either smoking cessation or smoking reduction in patients with schizophrenia, schizophreniform, schizoaffective, or delusional disorder. We excluded reviews, case series, case reports, comments, opinion, unpublished studies, conference posters, and abstracts only. We also excluded studies with overlapped data or data that could not be extracted. Three authors (SA, PVK, and RA) blindly screened all articles obtained through the search, based on titles and abstracts, to identify relevant articles for full-text consideration. Discrepancies were resolved by discussion, and consensus was achieved. Table 1 shows the list of studies considered in the final stage of selection, the decision about whether or not to include each relevant study, and the reasons for excluding the articles which were not chosen.

\section{Data Extraction}

Data were extracted by three blinded authors in order to preserve precision, using a template in Microsoft Excel to extract the needed data. The data that was extracted included sample size, age of subject, sex of subject, follow-up period, and drug dosage. Additionally, data for tested outcomes, the number of cigarettes

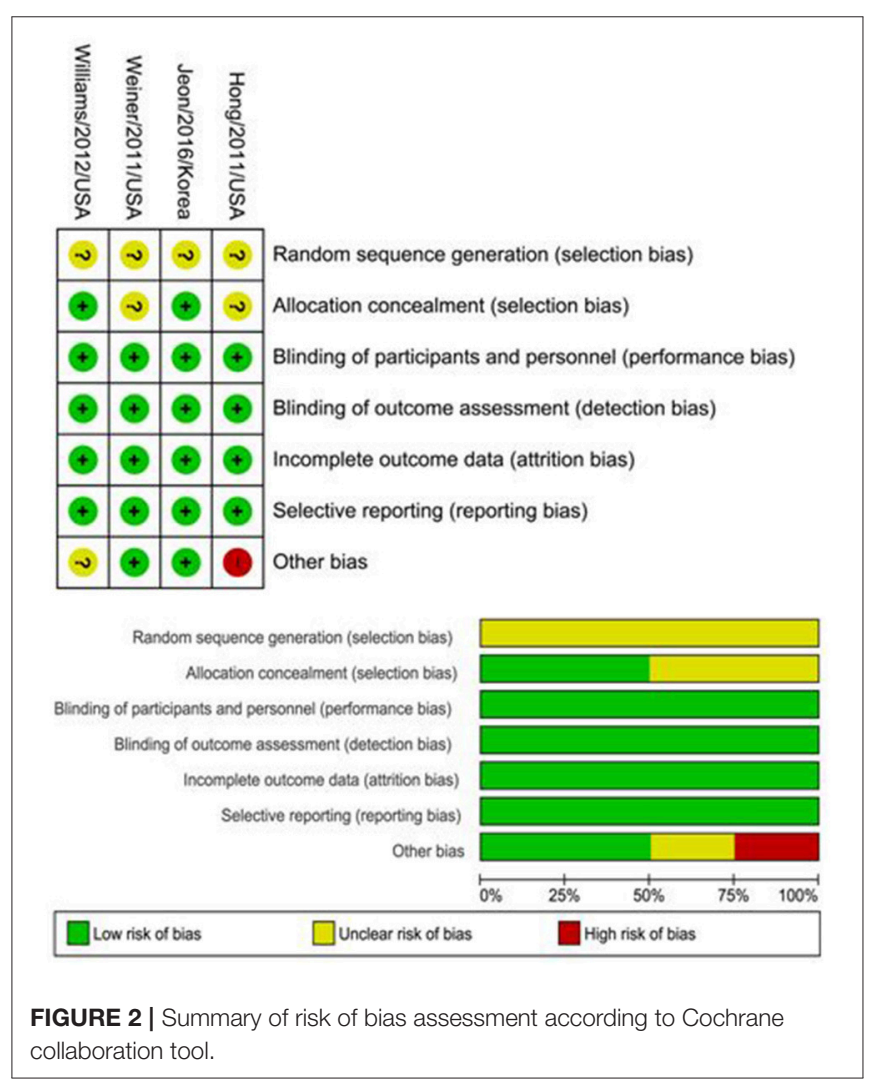

TABLE 2 | Characteristics of included studies.

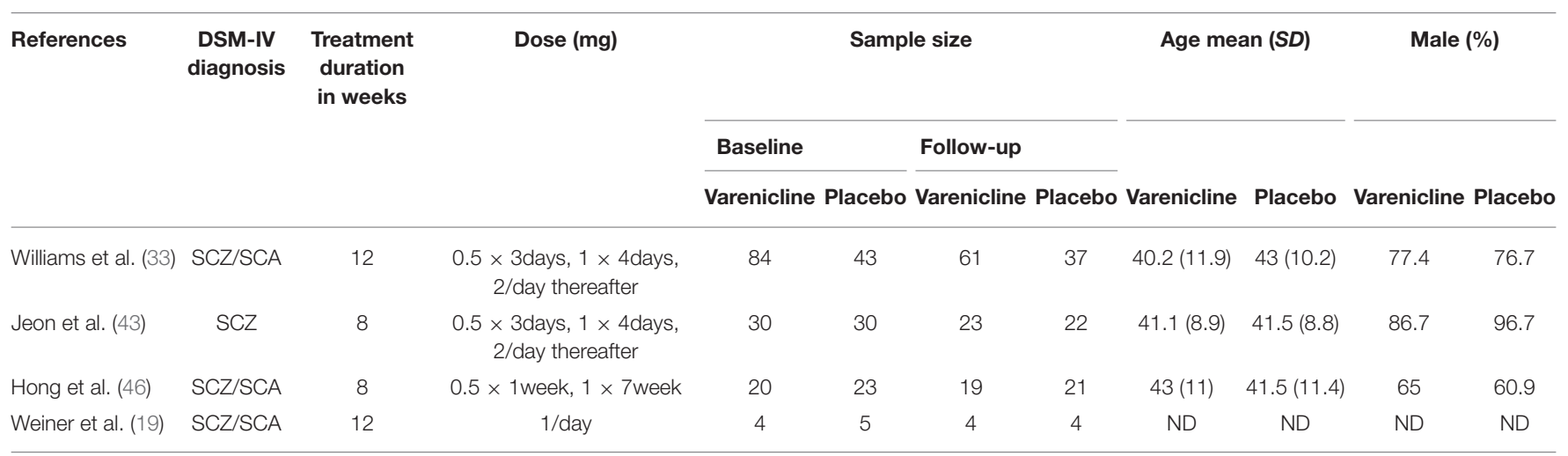

SD, standard deviation; SCZ, schizophrenia; SCA, schizoaffective; d, days. 
smoked per day, expired carbon monoxide, and abstinence from smoking behavior were extracted and meta-analyzed.

\section{Statistical Methods}

For dichotomous outcomes which are determined only at posttreatment, (such as the number of participants who remained abstinent), the log Odds Ratios (OR) and their respective sampling variances were calculated using the escalc function in the meta for package (47) in R. As for continuous outcomes studied both at baseline and post-treatment, the raw score, single group, pretest-posttest standardized mean difference (SMD) for both the treatment and placebo groups were computed separately (48). Their respective sampling variances were computed using formula A1 in (48). After this computation was complete, the placebo group's SMD was subtracted from that of the treatment group to obtain the overall SMD for a researched outcome in the study. The sampling variances of the treatment and placebo subgroups were then added together to obtain the overall sampling variance for that outcome in the study (48).

These SMDs were then pooled and meta-analyzed using random effects models (REM) to allow the effect sizes to vary across studies. The direction of the effect sizes was coded in such a way that larger positive effect sizes corresponded to larger positive differences (i.e., treatment-placebo) in raw scores. Heterogeneity was measured using the Q statistic; a significant Q statistic suggests that the variability among the effect sizes is larger than what is expected from subject sampling error alone. Follow-up leave-one-out analyses were carried out to assess the robustness of the results. These analyses were performed using the metafor package (47) in R 3.4.0.

\section{Quality Assessment}

The included studies were independently assessed using Cochrane collaboration's tool for assessing risk of bias (49). This tool is a two-part tool that addresses seven specific domains, including sequence generation, allocation concealment, blinding of participants, and personnel, blinding of outcome assessment, incomplete outcome data, selective outcome reporting, and other sources of bias. The judgment of each reviewer of each domain was categorized as "low risk," "high risk," or "unclear risk" of bias. Any disagreement was resolved by discussions between reviewers until a consensus was reached.

\section{RESULTS}

\section{Search Results}

The literature search yielded 822 articles. Five RCTs investigated the efficacy of varenicline in different clinical conditions and thus did not fulfill the core requirements of this meta-analysis. We summarize their interventions and the reasons for exclusion of these studies in Table $\mathbf{1}(9,32,42,45)$. The flowchart of studies' selection and screening is presented in Figure 1.

\section{Baseline Characteristics}

Our four included RCTs were comprised of 239 participants at baseline; however, only 191 completed the study and follow-up. The DSM-IV confirmed only schizophrenia in only one study (43), while schizophrenia or schizoaffective disorder was present in the participants in the remaining studies $(19,33,46)$. The participants' mean age ranged from 40 to 43 , and most of patients were male. In one of the studies, researchers did not indicate the age or gender of included participants (19). The treatment duration was 12 weeks in two studies $(19,33)$ and only 8 weeks in

TABLE 3 | Summary of leave-one-out analysis.

\begin{tabular}{lccc}
\hline Study removed & SMD & $\mathbf{9 5 \%}$ Cl & $\mathbf{Q}$ \\
\hline Williams et al. (33) & $0.71^{\star \star}$ & $0.26-1.16$ & $<0.001$ \\
Jeon et al. (43) & $0.97^{\star \star \star}$ & $0.59-1.36$ & 0.79 \\
Hong et al. (46) & $0.94^{\star \star \star}$ & $0.57-1.31$ & 1.01 \\
\hline
\end{tabular}

SMD, standardized mean difference; Cl, confidence intervals; ${ }^{* *} p<0.01 ;{ }^{* * *} p<0.001$.

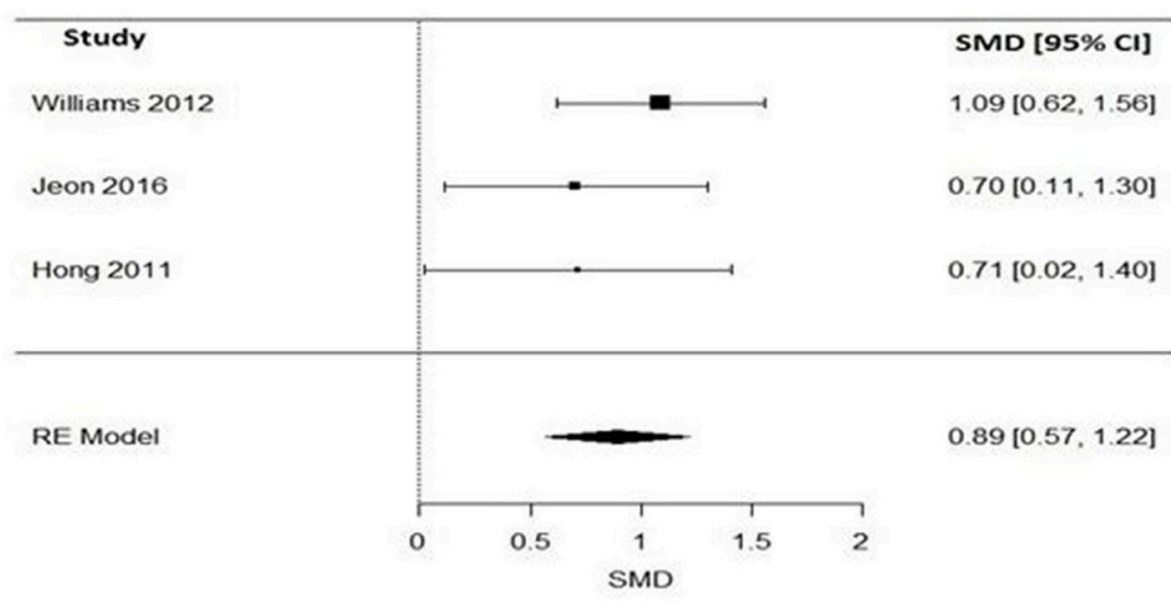

FIGURE 3 | Forest plot meta-analysis of three RCTs illustrating impact of varenicline compared to placebo on cigarette consumption in schizophrenia patients. SMD, standard mean difference; $\mathrm{Cl}$, confidence interval; $\mathrm{RE}$, random effect. 
the two remaining $(43,46)$. The characteristics of the studies are detailed in Table 2.

\section{Risk of Bias Among Included RCTs}

The random sequence generation presented an unclear risk of bias among the four included RCTs. Performance, detection, attrition, and reporting bias were low in all studies. Regarding allocation concealment, there was an unclear risk of bias in two studies $(19,46)$ while there was only a low risk in the remaining two $(33,43)$. The risk of bias in the studies is characterized in Figure 2.

\section{Outcomes}

\section{Impact of Varenicline on the Cigarette Consumption in Schizophrenia Patients}

Pooling together the three studies that evaluated the impact of varenicline on the number of cigarettes consumed per day, schizophrenic smokers who were given varenicline $(N$ $=134$ ) had significantly reduced the number of cigarettes consumed per day, when compared to those given a placebo $(N=94)$ (Figure 3). The adverse events among included RCTs are shown in Table 4. The Q statistic did not suggest significant heterogeneity in the random effects model (Table 5). Additionally, the leave-one-out analysis suggested that the obtained estimate was generally robust and that removal of any of the three studies did not alter the statistical significance (Table 3 ).

\section{Impact of Varenicline on the Expired Carbon Monoxide in Schizophrenia Patients}

Regarding levels of carbon monoxide, only two studies evaluated the impact of varenicline on the expired carbon monoxide levels before and after treatment $(43,46)$. Varenicline treatment $(N=49)$, compared to placebo $(N=51)$ significantly reduced the expired carbon monoxide levels in schizophrenia patients (Figure 4). Similarly, the Q statistic did not indicate significant heterogeneity in the random effects model (Table 5).
Impact of Varenicline on the Abstaining From Smoking Behavior in Schizophrenia Patients

Two studies have assessed the impact of varenicline on abstinence rates before and after treatment $(19,33)$. Again, vareniclinetreated schizophrenic patients $(N=87)$, relative to placebo group $(N=47)$, had significantly higher rates of abstaining from smoking behavior (Figure 5). The Q statistic did not indicate significant heterogeneity in the random effects model (Table 5).

\section{Adverse Events of Varenicline Compared to Placebo in Schizophrenic Patients}

The side effects reported in the included RCTs included gastrointestinal and psychiatric symptoms, as well as headache and fatigue. Nausea and insomnia were reported in three RCTs $(19,33,46)$, while vomiting, abdominal pain, abnormal dreams,

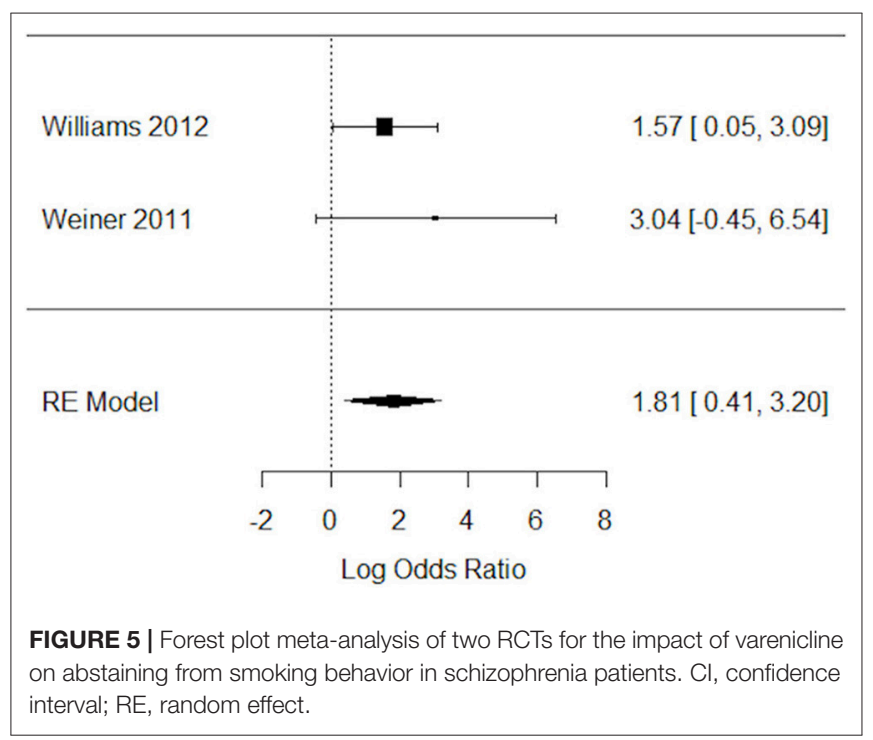

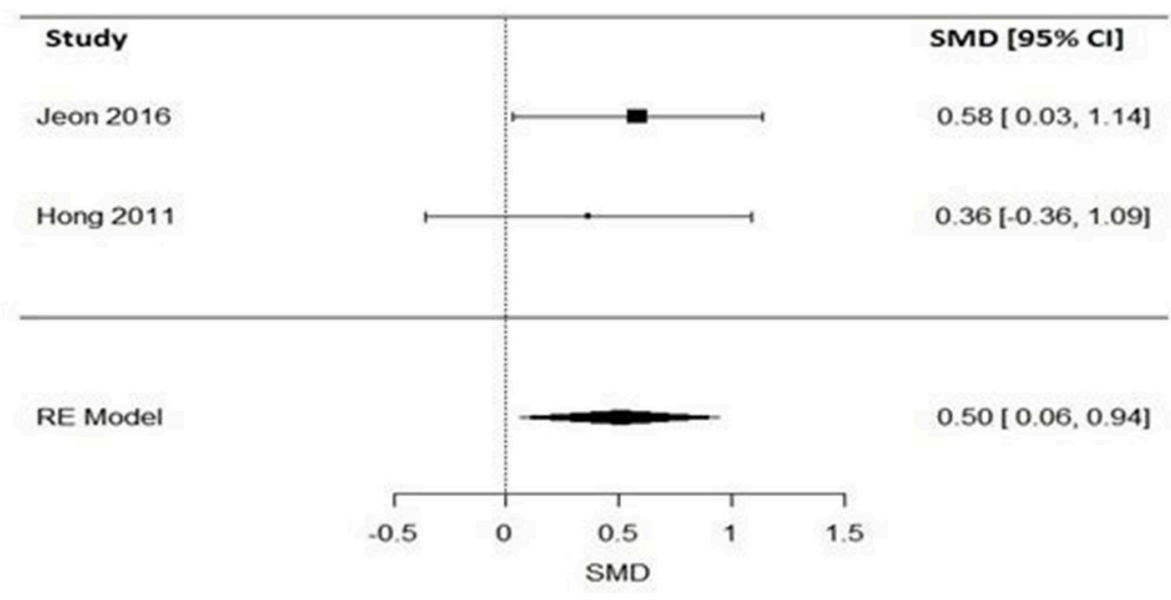

FIGURE 4 | Forest plot meta-analysis of two RCTs for the effect of varenicline compared to placebo on the expired carbon monoxide in schizophrenia patients. SMD, standard mean difference; $\mathrm{Cl}$, confidence interval; $\mathrm{RE}$, random effect. 


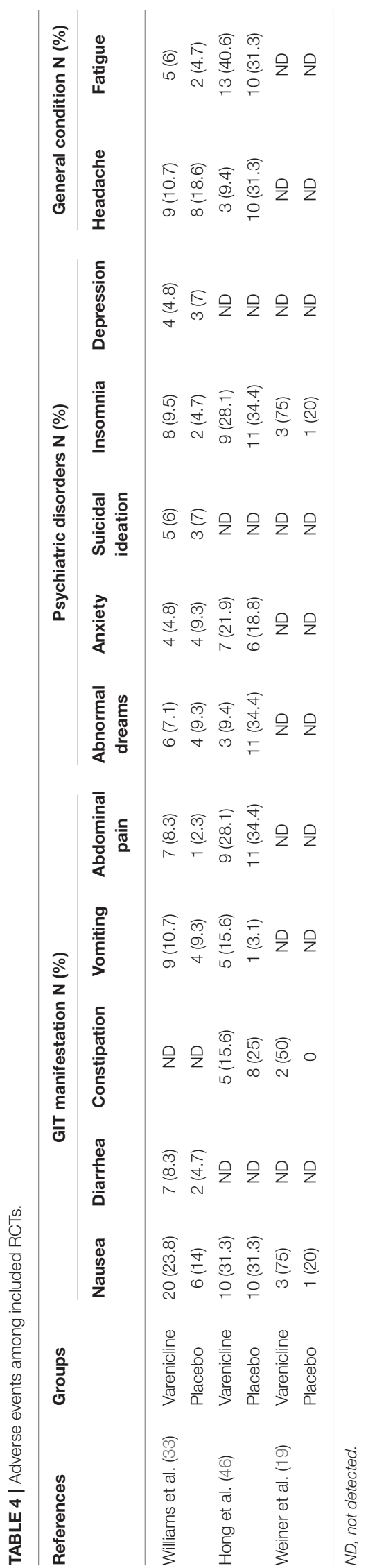

anxiety, headache, and fatigue were reported in two studies $(33,46)$. In only one study did some patients experience diarrhea and depression (33). Detailed information on reported adverse events are displayed in Table 4.

\section{DISCUSSION}

Varenicline has not been studied in patients with mental illnesses exclusively, particularly in patients with schizophrenia, a population with an exceptionally high prevalence of nicotine addiction. The safety and tolerability of varenicline has been debated and questioned, especially after initial case reports and small case series showed an increased risk of neuropsychiatric adverse effects $(23,24,50-53)$ in psychiatric populations. However, subsequent studies from case reports and case series to larger clinical trials $(9,15,19,31,33,37,42,43,45,46,53-$ 59) have demonstrated that the medication is well tolerated and safe to use to aid schizophrenia patients with smoking cessation.

Though the therapeutic potential of varenicline has been questioned in a recent meta-analysis (40), a thorough review of published studies in recent years provides adequate evidence that varenicline is an effective aid in smoking cessation in the schizophrenic population. A systematic review of the Cochrane database was conducted by (41) to evaluate the benefits and drawbacks of different treatments for nicotine dependence in schizophrenia. The authors found that smokers with schizophrenia who used varenicline were nearly five times more likely to abstain from smoking at the end of the treatment when compared to the placebo group. However, these results were obtained by analyzing a very small sample consisting of only two trials $(19,33)(2$ trials $N=137$, RR $4.74,95 \%$ CI 1.34 to 16.71 ).

Looking at more evidence from published trials such as Weiner et al. (19) studied varenicline for smoking cessation in eight patients with schizophrenia. In this RCT, the authors attempted to test the safety, tolerability, and efficacy of varenicline. The study determined varenicline to be safe and well-tolerated by schizophrenic patients (three of the four participants in the varenicline group were able to achieve sustained abstinence) and were not associated with any worsening of negative symptoms or other psychiatric symptoms as measured using BPRS scales. Overall, the side effects reported in the varenicline group included nausea, insomnia, constipation, all of which have been reported as side effects in the general population as well. Similarly, a 12-week randomized trial by William et al. (33) studied the efficacy of varenicline for smoking cessation in patients with stable schizophrenia or schizoaffective disorder. The study reported that at the end of treatment, 16 out of 84 in the varenicline group (19.0\%) met smoking-cessation criteria vs. 2 out of $43(4.7 \%)$ for the placebo group $(P=0.046)$. The study found varenicline to be a promising aid for smoking cessation, with higher rates of significance than placebo. Additionally, the study indicated that varenicline was well tolerated in schizophrenic patients for smoking cessation and 
TABLE 5 | Analysis summary of the parameters studied and their outcome.

\begin{tabular}{|c|c|c|c|c|c|c|}
\hline \multirow[t]{2}{*}{ Outcome } & \multirow[t]{2}{*}{ Varenicline $\mathbf{N}$} & \multirow[t]{2}{*}{ Placebo N } & \multicolumn{2}{|c|}{ Estimate } & \multirow[t]{2}{*}{$95 \% \mathrm{Cl}$} & \multirow[t]{2}{*}{$\mathbf{Q}$} \\
\hline & & & Type & Effect & & \\
\hline Cigarettes/day & 133 & 94 & SMD & $0.89^{\star \star \star}$ & $0.57,1.22$ & 1.33 \\
\hline Expired carbon monoxide & 49 & 51 & SMD & $0.50^{*}$ & $0.06,0.94$ & 0.22 \\
\hline Abstinence & 88 & 47 & Log OR & $1.81^{\star}$ & $0.41,3.20$ & 0.57 \\
\hline
\end{tabular}

N, number; SMD, standardized mean difference; OR, Odds Ratio; Cl, confidence intervals; ${ }^{*} p<0.05$; ${ }^{\star \star *} p<0.001$.

did not show any evidence of exacerbation of neuropsychiatric symptoms,

In 2015, a meta-analysis was conducted by Roberts et al. to assess the efficacy and tolerability of adjunctive pharmacotherapy for smoking cessation in patients with severe mental illnesses including schizophrenia, schizoaffective disorder, bipolar disorder, delusional disorder, and depressive psychoses (60). The authors analyzed published studies to examine the various smoking cessation pharmacotherapy modalities including nicotine replacement therapy (NRT), bupropion, and varenicline. The meta-analysis of a total of 17 studies suggested that bupropion and varenicline were more effective than placebo in a severely mentally ill population. A year later, another meta-analysis was conducted by $\mathrm{Wu}$ et al. (61) to determine the safety and effectiveness of varenicline in treating tobacco dependence in patients with severe mental illness, one of which was schizophrenia. This review analyzed eight randomized controlled trials in which the researchers compared varenicline with placebo or an alternative intervention for smoking cessation or reduction. The primary outcome measures of interest in the study were smoking cessation, measured by observing a change in the number of cigarettes smoked per day and the safety of varenicline smoked per day and the safety of varenicline, measured by noting the number of adverse psychiatric events. The study demonstrated that varenicline was significantly more effective than placebo, increasing the chance of success fourfold when compared to placebo; also, the reduction in smoking was greater in people with severe mental illness (SMI) when compared to the placebo group. The study defines SMI broadly to include non-organic disorders with psychotic features, schizophreniform and schizoaffective disorders, bipolar disorder, or delusional disorder. Although the study's results were found in favor of varenicline, the major limitation of this analysis was its inclusion of small-size trials, and the follow-up periods in all of the studies were short.

Kishi et al. (40) conducted a meta-analysis of randomized, double-blind, placebo-controlled trials (RCTs) to find the effects of varenicline adjuvant therapy for smoking cessation in people with schizophrenia. The results of the study suggested that, varenicline adjuvant therapy is not superior to placebo for smoking cessation in people with schizophrenia. Further, the study found that varenicline adjuvant therapy failed to show its superiority to placebo for positive, negative, and depressive symptoms. However, the study concluded that varenicline is well tolerated for smoking cessation in this population. Because of the many limitations of their metaanalysis including small-sample-size studies; the authors suggest further studies to examine varenicline on a larger scale with larger sample sizes. Nevertheless, the results of this metaanalysis came as a surprise to the psychiatric community, especially addiction psychiatrists. As a result of this study, Evins et al. (62) responded to this meta-analysis with a letter that highlighted the errors of the meta-analysis and mentioned that Kishi et al. (40) inappropriately included two trials which were not smoking cessation trials and that those trials were conducted on schizophrenic patients who had no intention of quitting. This caused the investigators to draw the erroneous conclusion that varenicline is ineffective for smoking cessation. Additionally, the meta-analysis inappropriately included a RTC trial by Shim et al. in which varenicline was examined as a cognitive enhancing agent (57). The meta-analysis also inappropriately included a relapse prevention trial of smoking cessation in schizophrenia patients (63). Although it is highly commendable that Kishi et al. (40) examined the effectiveness and safety of varenicline, a traditionally understudied topic. Because of analysis included inappropriate trial studies, the results of meta-analysis were opposite to numerous evidencedbased trials. Such error could lead readers to draw the wrong conclusions; therefore, the results could cause potential harm and lead to an underuse of this medication in this population.

Such erroneous findings as discussed above urged scientific community including authors of the current study to conduct an updated meta-analysis. Consequently, we did the metaanalysis using comprehensively defined inclusion and exclusion criteria. The results of our study show that varenicline is an effective and safe drug to use for smoking cessation in patients with schizophrenia. It is well tolerated and an effective aid in reducing cigarette consumption. The novelty of the current study is evidenced by results of the study, which were drawn after modifying and refining our analytic methodology. We expanded the parameters literature search, added recently published studies especially after 2015. Further, our study has a few strengths such as refining our inclusion criteria to target only studies that elucidated the efficacy and safety of varenicline for smoking cessation in schizophrenic patients. We did not consider any relapse prevention studies nor trials that examined the role of varenicline as a cognition-enhancing drug for smokers and nonsmokers; such an addition might have contaminated the 
sample and therefore lead to erroneous results. We assessed the quality of evidence by using the Cochrane tool, which is the universally accepted and recommended methodology for analysis of randomized trials. Moreover, compared to previously published meta-analysis, our electronic search for studies was carried out on five major databases, PubMed, Scopus, EMBASE, Web of Science, and Cochrane Library, a reasonably appropriate large search for performing a systematic review and metaanalysis.

Our study has suffered from limitations such as the small number of included participants; thus, our results should be implemented cautiously in clinical practice. The studies which we didn't include in the study are Dutra et al. (42), Pachas et al. (9), Fatemi et al. (2), Coles et al. (32) due to the lack of common variables and measures to assess final outcomes and the lack of relevant data from which we could extract information such as conference posters or abstracts only. Additionally, we excluded studies for which there was no control group (Table 1). Another limitation of our study was the unclear risk of bias regarding the selection of participants among all included RCTs. We also could not carry out subgroup meta-analysis or meta-regression due to the small number of included RCTs. We did not conduct pre- and post-meta-analysis for studies with no control group, and only those with a control arm were eligible for inclusion within our study.

\section{REFERENCES}

1. Yousefi MK, Folsom TD, Fatemi SH. A review of varenicline's efficacy and tolerability in smoking cessation studies in subjects with schizophrenia. $J$ Addict Res Ther. (2011) S4:001. doi: 10.4172/2155-6105.S4-001

2. Fatemi S, Stary J, Hatsukami D, Murphy S. A double-blind placebo-controlled cross over trial of bupropion in smoking reduction in schizophrenia. Schizophrenia Res. (2005) 76:353-6. doi: 10.1016/j.schres.2005.02.021

3. Weiner E, Ahmed S. Smoking cessation in schizophrenia. Curr Psychiatry Rev. (2013) 9:164-72. doi: 10.2174/1573400511309020011

4. Ahmed S, Reena MD, Nuzhat Hussain MD, Ahmed R, Amin S, Ahmed S, et al. Nicotine addiction in schizophrenia, availability of better treatment options as are in general population. Int J Emergency Ment Health Hum Resilience. (2014) 17:156-66.

5. de Leon J, Diaz FJ. A meta-analysis of worldwide studies demonstrates an association between schizophrenia and tobacco smoking behaviors. Schizophrenia Res. (2005) 76:135-57. doi: 10.1016/j.schres.2005.02.010

6. Tidey JW, Rohsenow DJ, Kaplan GB, Swift RM. Cigarette smoking topography in smokers with schizophrenia and matched non-psychiatric controls. Drug Alcohol Depend. (2005) 80:259-65. doi: 10.1016/j.drugalcdep.2005. 04.002

7. Williams JM, Gandhi KK, Lu S-E, Kumar S, Shen J, Foulds J, et al. Higher nicotine levels in schizophrenia compared with controls after smoking a single cigarette. Nicotine Tobacco Res. (2010) 12:855-9. doi: 10.1093/ntr/ ntq102

8. Dervaux A, Laqueille $\mathrm{X}$. Tabac et schizophrénie: aspects épidémiologiques et cliniques. Smoking and schizophrenia: epidemiological and clinical features. L'encephale (2008) 34:299-305. doi: 10.1016/j.encep.2007.04.003

9. Pachas GN, Cather C, Pratt SI, Hoeppner B, Nino J, Carlini SV, et al. Varenicline for smoking cessation in schizophrenia: safety and effectiveness in a 12-week open-label trial. J Dual Diagnosis (2012) 8:117-25. doi: 10.1080/15504263.2012.663675

10. Goff DC, Cather C, Evins AE, Henderson DC, Freudenreich O, Copeland $\mathrm{PM}$, et al. Medical morbidity and mortality in schizophrenia: guidelines for psychiatrists. J Clin Psychiatry (2005) 66:183-94. doi: 10.4088/JCP.v6 6 n0205

\section{CONCLUSION AND FUTURE PERSPECTIVES}

While the current meta-analysis demonstrates therapeutic efficacy of varenicline in promoting smoking cessation in clinically stable schizophrenic patients, however the concussion is subjected to certain limitations. Because of our well-established inclusion criteria and because of the relatively few clinical studies performed on this topic, we analyzed only a small number of studies. However, larger controlled trials on this topic are needed to draw firm and evidence based conclusion. Future clinical studies should consider assessing the impact of the duration of varenicline treatment, its role in relapse prevention over extended periods of time, dose dependent responses for smoking cessation, and consider smoking reduction in addition to smoking cessation.

\section{AUTHOR CONTRIBUTIONS}

SA, SV, VK, and MA: Study conception. SA, RA, AZ, SV, and JK: Study design. RB, AK, MQ, SA, and JK: Data acquisition. SA, AK, RA, AZ, MQ, and MA: Data analysis and interpretation. SA, SV, $A K, M Q$, and RB: Writing the paper draft. SA, MQ, RA, AZ, RB, and MA: Critical revision of the manuscript. GB: Interpretation, writing the paper draft, critical revision of the manuscript.

11. Hennekens $\mathrm{CH}$. Increasing global burden of cardiovascular disease in general populations and patients with schizophrenia. J Clin Psychiatry (2007) 68:4-7.

12. Saha S, Chant D, McGrath J. A systematic review of mortality in schizophrenia: is the differential mortality gap worsening over time? Arch Gen Psychiatry (2007) 64:1123-31. doi: 10.1001/archpsyc.64.1 0.1123

13. Baker A, Richmond R, Haile M, Lewin TJ, Carr VJ, Taylor RL, et al. A randomized controlled trial of a smoking cessation intervention among people with a psychotic disorder. Am J Psychiatry (2006) 163:1934-42. doi: 10.1176/ajp.2006.163.11.1934

14. Evins AE, Cather C, Deckersbach T, Freudenreich O, Culhane MA, Olm-Shipman CM, et al. A double-blind placebo-controlled trial of bupropion sustained-release for smoking cessation in schizophrenia. $J$ Clin Psychopharmacol. (2005) 25:218-25. doi: 10.1097/01.jcp.0000162802. 54076.18

15. Evins AE, Goff DC. Varenicline treatment for smokers with schizophrenia: a case series. J Clin Psychiatry (2008) 69:1016. doi: 10.4088/JCP.v69n 0620a

16. George TP, Krystal JH. Comorbidity of psychiatric and substance abuse disorders. Curr Opin Psychiatry (2000) 13:327-31. doi: 10.1097/00001504-200005000-00015

17. George TP, Vessicchio JC, Termine A, Bregartner TA, Feingold A, Rounsaville BJ, et al. A placebo controlled trial of bupropion for smoking cessation in schizophrenia. Biol Psychiatry (2002) 52:53-61. doi: 10.1016/S0006-3223(02)01339-2

18. George TP, Vessicchio JC, Sacco KA, Weinberger AH, Dudas MM, Allen TM, et al. A placebo-controlled trial of bupropion combined with nicotine patch for smoking cessation in schizophrenia. Biol Psychiatry (2008) 63:1092-6. doi: 10.1016/j.biopsych.2007.11.002

19. Weiner E, Buchholtz A, Coffay A, Liu F, McMahon RP, Buchanan RW, et al. Varenicline for smoking cessation in people with schizophrenia: a double blind randomized pilot study. Schizophrenia Res. (2011) 129:94-5. doi: 10.1016/j.schres.2011.02.003

20. Evins AE, Cather C, Culhane MA, Birnbaum A, Horowitz J, Hsieh E, et al. A 12-week double-blind, placebo-controlled study of bupropion sr added to high-dose dual nicotine replacement therapy for smoking cessation 
or reduction in schizophrenia. J Clin Psychopharmacol. (2007) 27:380-6. doi: 10.1097/01.jcp.0b013e3180ca86fa

21. Evins AE, Mays VK, Cather C, Goff DC, Rigotti NA, Tisdale T. A pilot trial of bupropion added to cognitive behavioral therapy for smoking cessation in schizophrenia. Nicotine Tobacco Res. (2001) 3:397-403. doi: 10.1080/14622200110073920

22. Weiner E, Ball MP, Summerfelt A, Gold J, Buchanan RW. Effects of sustained-release bupropion and supportive group therapy on cigarette consumption in patients with schizophrenia. Am J Psychiatry (2001) 158:6357. doi: 10.1176/appi.ajp.158.4.635

23. May AC, Rose D. Varenicline withdrawal-induced delirium with psychosis. Am J Psychiatry (2010) 167:720-1. doi: 10.1176/appi.ajp.2010.09081194

24. Kohen I, Kremen N. Varenicline-induced manic episode in a patient with bipolar disorder. Am J Psychiatry (2007) 164:1269-70. doi: 10.1176/appi.ajp.2007.07010173

25. Popkin MK. Exacerbation of recurrent depression as a result of treatment with varenicline. Am J Psychiatry (2008) 165:774. doi: 10.1176/appi.ajp.2008.07111735

26. Pumariega AJ, Nelson R, Rotenberg L. Varenicline-induced mixed mood and psychotic episode in a patient with a past history of depression. CNS Spectr. (2008) 13:511-4. doi: 10.1017/S1092852900016746

27. DiPaula BA, Thomas MD. Worsening psychosis induced by varenicline in a hospitalized psychiatric patient. Pharmacotherapy (2009) 29:852-7. doi: 10.1592/phco.29.7.852

28. Cinemre B, Akdag ST, Metin O, Doganavsargil O. Varenicline-induced psychosis. CNS Spectr. (2010) 15:470-2. doi: 10.1017/S1092852900000407

29. Food, Administration D, Alert FMW. Varenicline (Marketed as Chantix): Information. February 1, 2008, and November 20, (2007) Available online at: vww fda gov/cder/drug/infopage/varenicline/default htm (Accessed April 20, 2008).

30. Fatemi SH. Varenicline efficacy and tolerability in a subject with schizophrenia. Schizophrenia Res. (2008) 103:328-9. doi: 10.1016/j.schres.2008.05.002

31. Smith SS, McCarthy DE, Japuntich SJ, Christiansen B, Piper ME, Jorenby DE, et al. Comparative effectiveness of 5 smoking cessation pharmacotherapies in primary care clinics. Archives Internal Med. (2009) 169:2148-55. doi: 10.1001/archinternmed.2009.426

32. Coles A, Kozak K, Sharif-Razi M, McKee S, George T. 175. Effects of the nicotinic partial agonist varenicline on smoking lapse in smokers with and without schizophrenia. Biol Psychiatry (2017) 81:S73. doi: 10.1016/j.biopsych.2017.02.188

33. Williams JM, Anthenelli RM, Morris CD, Treadow J, Thompson JR, Yunis C, et al. A randomized, double-blind, placebo-controlled study evaluating the safety and efficacy of varenicline for smoking cessation in patients with schizophrenia or schizoaffective disorder". J Clin Psychiatry.(2012) 73:654-60. doi: 10.4088/JCP.11m 07522

34. Gonzales D, Rennard SI, Nides M, Oncken C, Azoulay S, Billing CB, et al. Varenicline, an $\alpha 4 \beta 2$ nicotinic acetylcholine receptor partial agonist, vs sustained-release bupropion and placebo for smoking cessation: a randomized controlled trial. JAMA (2006) 296:47-55. doi: 10.1001/jama.296.1.47

35. Jorenby DE, Hays JT, Rigotti NA, Azoulay S, Watsky EJ, Williams KE, et al. Efficacy of varenicline, an $\alpha 4 \beta 2$ nicotinic acetylcholine receptor partial agonist, vs placebo or sustained-release bupropion for smoking cessation: a randomized controlled trial. JAMA (2006) 296:56-63. doi: $10.1001 /$ jama.296.1.56

36. Oncken C, Gonzales D, Nides M, Rennard S, Watsky E, Billing CB, et al. Efficacy and safety of the novel selective nicotinic acetylcholine receptor partial agonist, varenicline, for smoking cessation. Arch Internal Med. (2006) 166:1571-7. doi: 10.1001/archinte.166.15.1571

37. Stapleton JA, Watson L, Spirling LI, Smith R, Milbrandt A, Ratcliffe $\mathrm{M}$, et al. Varenicline in the routine treatment of tobacco dependence: a pre-post comparison with nicotine replacement therapy and an evaluation in those with mental illness. Addiction (2008) 103:146-54. doi: 10.1111/j.1360-0443.2007.02083.x

38. Tsai ST, Cho HJ, Cheng HS, Kim CH, Hsueh KC, Billing CB, et al. A randomized, placebo-controlled trial of varenicline, a selective $\alpha 4 \beta 2$ nicotinic acetylcholine receptor partial agonist, as a new therapy for smoking cessation in Asian smokers. Clin Ther. (2007) 29:1027-39. doi: 10.1016/j.clinthera.2007.06.011

39. Wang C, Xiao D, Chan KPW, Pothirat C, Garza D, Davies S. Varenicline for smoking cessation: a placebo-controlled, randomized study. Respirology (2009) 14:384-92. doi: 10.1111/j.1440-1843.2008.01476.x

40. Kishi T, Iwata N. Varenicline for smoking cessation in people with schizophrenia: systematic review and meta-analysis. Eur Arch Psychiatry Clin Neurosci. (2015) 265:259-68. doi: 10.1007/s00406-014-0551-3

41. Tsoi DT, Porwal M, Webster AC. Interventions for smoking cessation and reduction in individuals with schizophrenia. Cochrane Database Syst Rev. (2013) CD007253. doi: 10.1002/14651858.CD007253.pub3

42. Dutra SJ, Stoeckel LE, Carlini SV, Pizzagalli DA, Evins AE. Varenicline as a smoking cessation aid in schizophrenia: effects on smoking behavior and reward sensitivity. Psychopharmacology (2012) 219:25-34. doi: 10.1007/s00213-011-2373-6

43. Jeon DW, Shim JC, Kong BG, Moon JJ, Seo YS, Kim SJ, et al. Adjunctive varenicline treatment for smoking reduction in patients with schizophrenia: a randomized double-blind placebo-controlled trial. Schizophrenia Res. (2016) 176:206-11. doi: 10.1016/j.schres.2016.08.016

44. Moher D, Liberati A, Tetzlaff J, Altman DG, Group P. Preferred reporting items for systematic reviews and meta-analyses: the PRISMA statement. PLoS Med. (2009) 6:e1000097. doi: 10.1371/journal.pmed.10 00097

45. Fatemi SH, Yousefi MK, Kneeland RE, Liesch SB, Folsom TD, Thuras PD. Antismoking and potential antipsychotic effects of varenicline in subjects with schizophrenia or schizoaffective disorder: a double-blind placebo and bupropion-controlled study. Schizophrenia Res. (2013) 146:3768. doi: 10.1016/j.schres.2013.02.015

46. Hong LE, Thaker GK, McMahon RP, Summerfelt A, RachBeisel J, Fuller RL, et al. Effects of moderate-dose treatment with varenicline on neurobiological and cognitive biomarkers in smokers and nonsmokers with schizophrenia or schizoaffective disorder. Arch Gen Psychiatry (2011) 68:1195-206. doi: 10.1001/archgenpsychiatry.2011.83

47. Viechtbauer W. Conducting meta-analyses in R with the metafor package. $J$ Stat Softw. (2010) 36:1-48. doi: 10.18637/jss.v036.i03

48. Morris SB, DeShon RP. Combining effect size estimates in meta-analysis with repeated measures and independent-groups designs. Psychol Methods (2002) 7:105. doi: 10.1037/1082-989X.7.1.105

49. Higgins JP, Altman DG, Gøtzsche PC, Jüni P, Moher D, Oxman AD, et al. The cochrane collaboration's tool for assessing risk of bias in randomised trials. BMJ (2011) 343:d5928. doi: 10.1136/bmj.d5928

50. Freedman R. Exacerbation of schizophrenia by varenicline. Am J Psychiatry (2007) 164:1269. doi: 10.1176/appi.ajp.2007.07020326

51. Moore TJ, Glenmullen J, Furberg CD. Thoughts and acts of aggression/violence toward others reported in association with varenicline. Ann Pharmacother. (2010) 44:1389-94. doi: 10.1345/aph.1P172

52. Ismail Z, Syms J, Blumberger D, George TP. Varenicline induced polydipsia and hyponatremia in a patient with schizophrenia. Schizophrenia Res. (2010) 119:268. doi: 10.1016/j.schres.2010.01.027

53. Waldo MC, Woodward L, Adler LE. Varenicline and P50 auditory gating in medicated schizophrenic patients: a pilot study. Psychiatry Res. (2010) 175:179-80. doi: 10.1016/j.psychres.2009.0 1.025

54. Grosshans M, Mutschler J, Hermann D, Mann K, Diehl A. Reduced affective symptoms during tobacco dependence treatment with varenicline. Addiction (2009) 104:859-61. doi: 10.1111/j.1360-0443.2009.02537.x

55. Liu ME, Tsai SJ, Jeang SY, Peng SL, Wu SL, Chen MC, et al. Varenicline prevents affective and cognitive exacerbation during smoking abstinence in male patients with schizophrenia. Psychiatry Res. (2011) 190:79-84. doi: 10.1016/j.psychres.2011.04.018

56. McClure JB, Swan GE, Catz SL, Jack L, Javitz H, McAfee T, et al. Smoking outcome by psychiatric history after behavioral and varenicline treatment. $J$ Subst Abuse Treat. (2010) 38:394-402. doi: 10.1016/j.jsat.2010.03.007

57. Shim JC, Jung DU, Jung SS, Seo YS, Cho DM, Lee JH, et al. Adjunctive varenicline treatment with antipsychotic medications for cognitive impairments in people with schizophrenia: a randomized double-blind placebo-controlled trial. Neuropsychopharmacology (2012) 37:660-8. doi: $10.1038 /$ npp. 2011.238 
58. Purvis TL, Mambourg SE, Balvanz TM, Magallon HE, Pham RH. Safety and effectiveness of varenicline in a veteran population with a high prevalence of mental illness. Ann Pharmacother. (2009) 43:862-7. doi: 10.1345/aph. 1L661

59. Anghelescu I. Successful smoking cessation and improvement of negative symptoms with varenicline in a stable schizophrenia patient. $J$ Neuropsychiatry Clin Neurosci. (2009) 21:102-3. doi: 10.1176/jnp.2009. 21.1.102

60. Roberts E, Eden Evins A, McNeill A, Robson D. Efficacy and tolerability of pharmacotherapy for smoking cessation in adults with serious mental illness: a systematic review and network meta-analysis. Addiction (2016) 111:599-612. doi: 10.1111 /add.13236

61. Wu Q, Gilbody S, Peckham E, Brabyn S, Parrott S. Varenicline for smoking cessation and reduction in people with severe mental illnesses: systematic review and meta-analysis. Addiction (2016) 111:1554-67. doi: 10.1111/add.13415

62. Evins AE, Hong LE, Kelly DL. T. Kishi and N. Iwata: varenicline for smoking cessation in people with schizophrenia: systematic review meta-analysis. Eur Arch Psychiatry Clin Neurosci. (2015) 265:269-70. doi: 10.1007/s00406-014-0556-y

63. Evins AE, Cather C, Pratt SA, Pachas GN, Hoeppner SS, Goff DC, et al. Maintenance treatment with varenicline for smoking cessation in patients with schizophrenia and bipolar disorder: a randomized clinical trial. JAMA (2014) 311:145-54. doi: 10.1001/jama.2013.285113

Conflict of Interest Statement: The authors declare that the research was conducted in the absence of any commercial or financial relationships that could be construed as a potential conflict of interest.

Copyright (c) 2018 Ahmed, Virani, Kotapati, Bachu, Adnan, Khan, Zubair, Begum, Kumar, Qureshi and Ahmed. This is an open-access article distributed under the terms of the Creative Commons Attribution License (CC BY). The use, distribution or reproduction in other forums is permitted, provided the original author(s) and the copyright owner(s) are credited and that the original publication in this journal is cited, in accordance with accepted academic practice. No use, distribution or reproduction is permitted which does not comply with these terms. 